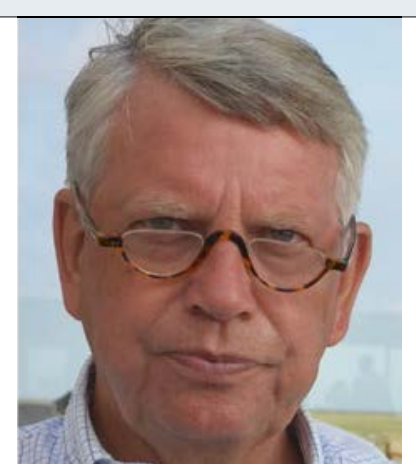

Prof. Dr. Gerald Klose

Praxis Dres.

T. Beckenbauer \&

S. Maierhof, Bremen

\title{
Therapie-Highlights in der Angiologie und Lipidologie
}

$\mathrm{N}$ eue Therapiemöglichkeiten nach Thrombose und Lungenembolie zeigen C. Regli und M. Heizmann auf. Die Mehrzahl betroffener Patienten wird inzwischen mit direkten oralen Antikoagulanzien (DOAK) behandelt, die in internationalen Leitlinien bereits als Mittel erster Wahl propagiert werden. Auch hat sich gezeigt, dass eine Langzeitprophylaxe mit DOAK bei Dosishalbierung nach 6 Monaten gleiche Effekte bei deutlich vermindertem Blutungsrisiko zeigt wie bei einer therapeutischen Dosierung.

Zwar sind die Amputationszahlen bei kritischer Extremitätenischämie mit und ohne Diabetes in Deutschland rückläufig, es wird aber noch deutlich mehr amputiert als z. B. in Skandinavien oder den Niederlanden. Betroffene Patienten sollten immer in einem kompetenten Gefäßzentrum betreut werden, fordern H. Lawall, W. Tigges und R. Lobmann. Das Einholen einer zweiten Meinung zur Klärung einer möglichen Revaskularisation hilft den Patienten: Die Zahl der Amputationen nimmt dann deutlich ab. Fächerübergreifende interdisziplinäre Kolloquien sind gefragt, was bei komplexen distalen arteriellen Durchblutungsstörungen und gleichzeitigem diabetischen Fußsyndrom besonders deutlich wird.

Ein wesentlicher Risikofaktor für die Entstehung einer peripheren arteriellen Verschlusskrankheit (PAVK) ist das Lebensalter. Während junge Patienten meist "nur" eine Claudicatio intermittens aufweisen, kommen bei den Betagten erschwerend weitere Symptomenkomplexe hinzu. Das stellt eine besondere Herausforderung für den Angiologen dar, wie C. Ploenes darlegt. Es ist sehr erfreulich, dass dieses Kapitel mit geriatrischen und angiologischen Aspekten auch in den neuen S-3 AWMF-Leitlinien zum Management der PAVK seine Berücksichtigung gefunden hat.

Die Publikation der neuen Dyslipidämie-Leitlinien der European Society of Cardiology und der European Atherosclerosis Society gehört zu den diesjährigen Höhepunkten klinischer Lipidologie. Im aktuellen
Schwerpunkt wird dargestellt, in welchem Umfang die auf den ersten Blick gering erscheinenden Änderungen den präventivmedizinischen Anspruch lipidwirksamer Therapie deutlich verschärfen. Dies führte - zu Recht - zum Überschreiten eines ausschließlich auf RCT-Evidenz basierenden Konsens, weil bei der Patientenversorgung Fragen auftauchen, für deren Beantwortung auch weitere Formen von Evidenz erforderlich sind. Hierzu gehören besonders die Bewertung von Lp(a)-Erhöhungen und Überlegungen, bis zu welchem Ausmaß die heute erreichbaren sehr niedrigen LDL-Cholesterin-Konzentrationen sicher sind.

Die in den o.g. Leitlinien kaum gestreiften klinischen Implikationen erhöhter Lp(a)-Konzentrationen werden im Beitrag von I. Gouni Berthold auf der Basis der in unvergleichbar großem Umfang berücksichtigten zugehörigen wissenschaftlichen Literatur analysiert. Danach kann es keine andere Schlussfolgerung geben als die Forderung randomisierter, kontrollierter Studien mit der Fragestellung, ob die selektive Reduktion von erhöhten Lp(a)-Konzentrationen in der Lage ist, kardiovaskuläre Endpunkte positiv zu beeinflussen. Vorerst muss bei Höchstrisiko-Patienten die Lipoprotein-Apherese eine therapeutische Option bleiben.

H. Leitholf arbeitet zum Thema Sicherheit sehr niedriger LDL-Cholesterin-Konzentrationen fundiert mögliche Antworten auf der Basis unterschiedlicher Pathophysiologie genetischer Hypolipoproteinämien und bislang fehlender Unsicherheitssignale therapeutisch erreichter LDL-Cholesterin-Absenkungen heraus. Die zur Zeit der Beitragserstellung noch nicht veröffentlichte mit Evolocumab durchgeführte GLAGOV-Studie (S.13) bestätigte die Sicherheit mittels PCSK9-Inhibitor erreichter sehr niedriger LDLCholesterin-Konzentrationen - ein weiteres Highlight der Lipidologie in 2016.

Ihre

Curt Diehm und Gerald Klose 